\title{
Persistent current of correlated electrons in mesoscopic ring with impurity
}

\author{
R. Krčmár a , A. Gendiar ${ }^{\text {a }}$, M. Moško ${ }^{a}$, R. Németh ${ }^{\mathrm{a}}, \mathrm{P}$. Vagner $^{\mathrm{a}}$, L. Mitas ${ }^{\mathrm{b}}$ \\ ${ }^{a}$ Institute of Electrical Engineering, Centre of Excellence CENG, Slovak Academy of Sciences, 84104 Bratislava, Slovakia \\ b Department of Physics, North Carolina State University, Raleigh, NC 27695
}

\begin{abstract}
The persistent current of correlated electrons in a continuous one-dimensional ring with a single scatterer is calculated by solving the many-body Schrodinger equation for several tens of electrons interacting via the electron-electron (e-e) interaction of finite range. The problem is solved by the configuration-interaction (CI) and diffusion Monte Carlo (DMC) methods. The CI and DMC results are in good agreement. In both cases, the persistent current $I$ as a function of the ring length $L$ exhibits the asymptotic dependence $I \propto L^{-1-\alpha}$ typical of the Luttinger liquid, where the power $\alpha$ depends only on the e-e interaction. The numerical values of $\alpha$ agree with the known formula of the renormalisation-group theory.
\end{abstract}

Key words: one-dimensional transport, mesoscopic ring, persistent current, electron-electron interaction PACS: 73.23.-b, 73.61.Ey

A one-dimensional (1D) interacting electron system cannot be viewed as a Fermi liquid of non-interacting quasi-particles. Away from the charge-density-wave instability, the interacting 1D system is a Luttinger liquid in which the elementary excitations are of collective Bosonic nature (see the review [1]). The Luttinger liquid properties affect the $1 \mathrm{D}$ electron transport. An interesting example of such effect is the persistent electron current in a mesoscopic $1 \mathrm{D}$ ring. Magnetic flux $\phi$ piercing the mesoscopic ring gives rise to the persistent current which can be expressed (at $T=0 \mathrm{~K}$ ) as [2]

$$
I=-\partial E_{0}(\phi) / \partial \phi
$$

where $E_{0}$ is the eigenenergy of the many-body groundstate. If the ring is clean and the single-particle dispersion law is parabolic, the electron-electron (e-e) interaction does not affect the persistent current owing to the Galilean invariance of the problem. However, if

Email address: roman.krcmar@savba.sk (R. Krčmár).

Preprint submitted to Physica E a single scatterer (an impurity or a weak link) is introduced into the ring, the non-interacting and interacting result are expected to differ fundamentally. For non-interacting spinless electrons in the 1D ring containing a single scatterer with transmission probability $\left|\tilde{t}_{k_{F}}\right|^{2} \ll 1$, the resulting persistent current depends on the magnetic flux and ring length $(L)$ as [3]

$$
I=\left(e v_{F} / 2 L\right)\left|\tilde{t}_{k_{F}}\right| \sin \left(\phi^{\prime}\right),
$$

where $\phi^{\prime} \equiv 2 \pi \phi / \phi_{0}, \phi_{0}=h / e$ is the flux quantum, $k_{F}$ is the Fermi wave vector, and $v_{F}$ is the Fermi velocity. For a spinless Luttinger liquid the persistent current follows the power law $I \propto L^{-\alpha-1}$. More precisely, [3]

$$
I \propto L^{-\alpha-1} \sin \left(\phi^{\prime}\right),
$$

where the power $\alpha$ depends only on the e-e interaction, not on the properties of the scatterer. The formula (3) can also be obtained [3] heuristically as follows. Matveev et al. [4] analyzed, how the e-e interaction renormalizes the bare transmission amplitude 
$\tilde{t}_{k_{F}}$ of a single scatterer in the middle of the $1 \mathrm{D}$ wire. They derived the renormalized amplitude $t_{k_{F}}$ by using the renormalization-group ( $\mathrm{RG}$ ) approach suitable for a weakly-interacting electron gas $(\alpha \ll 1)$. In the limit $\left|\tilde{t}_{k_{F}}\right|^{2} \ll 1$, their result can be expressed in the form

$$
t_{k_{F}} \simeq \tilde{t}_{k_{F}}(d / L)^{\alpha},
$$

where $L$ is the wire length, $d$ is the spatial range of the pair e-e interaction $V\left(x-x^{\prime}\right)$, and the power $\alpha$ is given (for spinless electrons) by expression

$$
\alpha=\left[V(0)-V\left(2 k_{F}\right)\right] / 2 \pi \hbar v_{F},
$$

with $V(q)$ being the Fourier transform of $V\left(x-x^{\prime}\right)$. If one replaces in equation (2) the bare $\tilde{t}_{k_{F}}$ by the renormalized amplitude (4), one recovers the Luttingerliquid dependence (3). This approach is heuristic and single-particle, nevertheless, $\alpha$ is now expressed microscopically in the form (5), expected to hold for $\alpha \ll 1$.

In the Luttinger-liquid model, the physics of the lowenergy excitations is mapped onto an effective field theory using Bosonization [3], where terms expected to be negligible at low energies are omitted. Within this model, the asymptotic dependence (3) was obtained by another approximation, using the analogy to the problem of quantum coherence in dissipative environment [3]. It would thus be useful to avoid this approximation as well as Bosonisation. This has been done in Ref. [5], where the persistent current was calculated numerically by solving the $1 \mathrm{D}$ lattice model with nearestneighbor hopping and interaction. The formula (3) was confirmed for long chains and strong scatterers.

In this work the persistent current of correlated electrons is studied in a continuous 1D ring, not in the 1D lattice. Using the configuration-interaction (CI) and diffusion Monte Carlo (DMC) methods, we solve the continuous many-body Schrodinger equation for several tens of electrons interacting via the e-e interaction

$$
V\left(x-x^{\prime}\right)=V_{0} \exp \left(-\left|x-x^{\prime}\right| / d\right) .
$$

Interaction (6) emulates screening and allows comparison with correlated models $[3,4,5]$ which also assume the e-e interaction of finite range. The figures 1 and 2 present our major CI and DMC results. These results show the persistent current exhibiting the Luttingerliquid law $I \propto L^{-1-\alpha}$, already verified by the lattice model [5]. However, it has sofar not been verified, that the power $\alpha$ obeys the RG formula (5) also in the microscopic model avoiding the RG approach. The CI and DMC data in figures 1 and 2 verify this fact quite well.

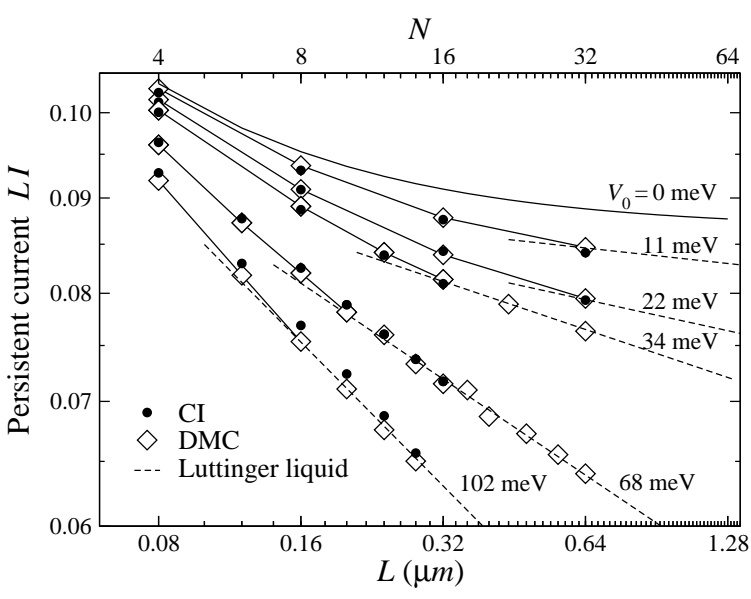

Fig. 1. Persistent current $L I\left(\phi=0.25 \phi_{0}\right)$ in the GaAs ring with a single scatterer as a function of the ring length $L$. The upper horizontal axis shows the number of electrons $N$, the electron density is $N / L=5 \times 10^{7} \mathrm{~m}^{-1}$. The transmission of the scatterer is $\left|\tilde{t}_{k_{F}}\right|^{2}=0.03$. The range of the e-e interaction is $d=3 \mathrm{~nm}$, the magnitude $V_{0}$ is varied. The CI and DMC data are shown by symbols. The full lines connecting the DMC data are a guide for eye. The dashed lines show the Luttinger liquid asymptotics $L I \propto L^{-\alpha}$, with $\alpha$ obtained from the RG formula (5): $\alpha=0.0277,0.0561,0.0855,0.1710$, and 0.2565 for $V_{0}=11,22,34,68$, and $102 \mathrm{meV}$, respectively.

We consider $N$ electrons with free motion along a circular $1 \mathrm{D}$ ring threaded by magnetic flux $\phi$ due to the constant magnetic field. The non-interacting singleelectron states $\varphi_{i}(x)$ obey the Schrödinger equation

$$
\left(-\frac{\hbar^{2}}{2 m} \frac{d^{2}}{d x^{2}}+\gamma \delta(x)\right) \varphi_{i}(x)=\epsilon_{i} \varphi_{i}(x),
$$

with boundary condition $\varphi_{i}(x+L)=\exp \left(i \frac{2 \pi \phi}{\phi_{0}}\right) \varphi_{i}(x)$, where $x$ is the electron coordinate along the ring, $m$ is the electron effective mass, and $\gamma \delta(x)$ is the potential barrier due to the scatterer. The wave functions $\varphi_{i}(x)$ and energy levels $\epsilon_{i}$ are found numerically as in Ref. [6]. We want to solve the Schrödinger equation $H \Psi=E \Psi$ for $N$ interacting electrons with Hamiltonian

$$
H=\sum_{i=1}^{N}\left(-\frac{\hbar^{2}}{2 m} \frac{d^{2}}{d x_{i}^{2}}+\gamma \delta\left(x_{i}\right)\right)+\frac{1}{2} \sum_{i \neq j} V\left(x_{i}-x_{j}\right) .
$$

Consider first the non-interacting many-body problem $\mathcal{H} \chi=\mathcal{E}_{\chi}$, where $\mathcal{H}$ is Hamiltonian (8) without the e-e interaction. Clearly, in this case $\mathcal{E}_{i}=\epsilon_{i_{1}}+\cdots+\epsilon_{i_{N}}$ and

$$
\chi_{i}=\frac{1}{\sqrt{N}}\left|\begin{array}{ccc}
\varphi_{i_{1}}\left(x_{1}\right) & \ldots & \varphi_{i_{N}}\left(x_{1}\right) \\
\vdots & \ddots & \vdots \\
\varphi_{i_{1}}\left(x_{N}\right) & \ldots & \varphi_{i_{N}}\left(x_{N}\right)
\end{array}\right|
$$




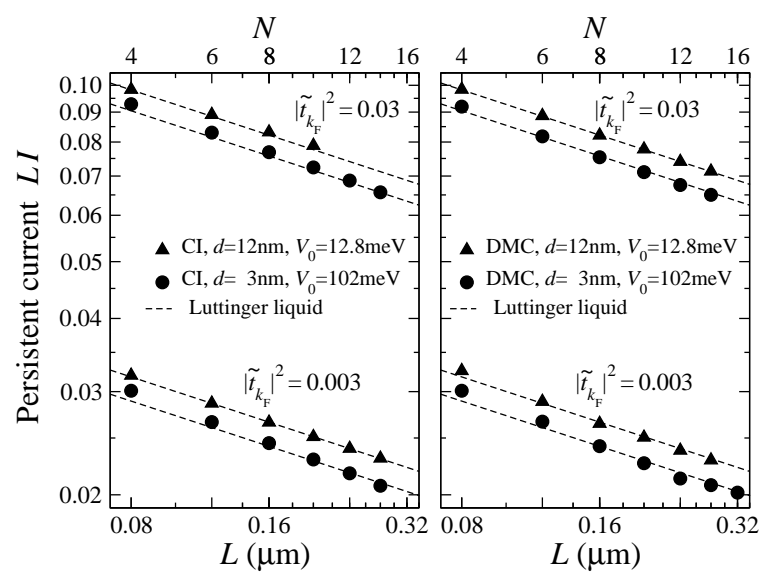

Fig. 2. Persistent current $L I\left(\phi=0.25 \phi_{0}\right)$ in the GaAs ring with a single scatterer as a function of the ring size. The parameters of the calculation are given in the figure. The $\mathrm{CI}$ and DMC data are shown by symbols. The dashed lines show the decay $L I \propto L^{-\alpha}$, with $\alpha$ given by the RG formula (5): $\alpha=0.2565$ for $d=3 \mathrm{~nm}$ and $V_{0}=102 \mathrm{meV}$ as well as for $d=12 \mathrm{~nm}$ and $V_{0}=12.8 \mathrm{meV}$. The CI and DMC data confirm this $\alpha$. They also confirm that $\alpha$ remains unchanged when the transmission $\left|\tilde{t}_{k_{F}}\right|^{2}$ of the scatterer is varied.

where $i$ is the quantum number labeling a specific set of $N$ occupied levels $\epsilon_{i_{1}}, \ldots, \epsilon_{i_{N}}$. The CI method [7] relies on expansion $\Psi=c_{0} \chi_{0}+c_{1} \chi_{1}+c_{2} \chi_{2}+\ldots$ Using this expansion and equation $\left\langle\chi_{i}|H| \Psi\right\rangle=\left\langle\chi_{i}|E| \Psi\right\rangle$ we get

$$
\sum_{j=0}^{\infty}\left(\mathcal{E}_{j} \delta_{i j}+V_{i j}\right) c_{j}=E c_{i}, \quad i=0,1, \ldots, \infty
$$

where $V_{i j}=\frac{1}{2}\left\langle\chi_{i}\left|\sum V\left(x_{k}-x_{l}\right)\right| \chi_{j}\right\rangle$. We reduce the infinite number of single-energy levels $\epsilon_{j}$ to the finite one by introducing a proper upper energy cutoff. This reduces the infinite number of equations (10) to a certain finite number $M+1$. We get the finite system $\sum_{j=0}^{M}\left(\mathcal{E}_{j} \delta_{i j}+V_{i j}\right) c_{j}=E c_{i}$, where $i=0,1, \ldots, M$. This system determines the eigenvalues $E_{l}$ and eigenvectors $\left(c_{0}^{l}, c_{1}^{l}, \ldots, c_{M}^{l}\right)$ for $l=0,1, \ldots, M$. To obtain the persistent current (1), we need only the groundstate energy $E_{l=0}$ as a function of $\phi$. We have obtained $E_{0}$ by solving the system with program ARPACK.

We have also calculated $E_{0}$ by the DMC method [7]. Technical details of this calculation are the same as in Ref. [8], the persistent current is obtained from (1).

Our major CI and DMC results are shown in figures 1 and 2. These figures show in log scale the persistent current $L I\left(\phi=0.25 \phi_{0}\right)$ as a function of the ring length for a GaAs ring with one strong scatterer. In log scale

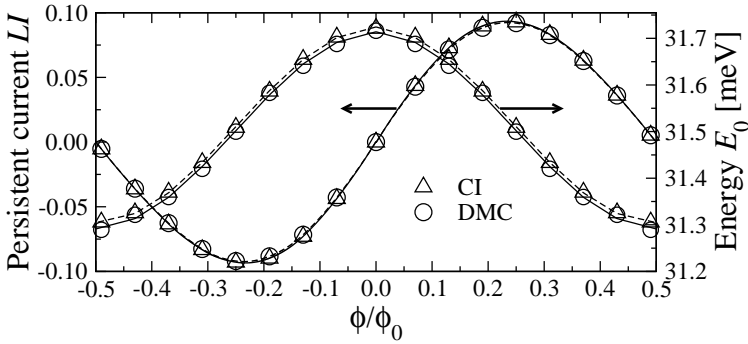

Fig. 3. Persistent current versus magnetic flux and energy of the many-body ground state for the GaAs ring with parameters $N=4$ and $L=0.08 \mu \mathrm{m}$. The transmission of the scatterer is set to $\left|\tilde{t}_{k_{F}}\right|^{2}=0.03$. The parameters of the e-e interaction are $V_{0}=102 \mathrm{meV}$ and $d=3 \mathrm{~nm}$. The CI and DMC data are shown by open symbols, the lines connecting points are a guide for eye. The data for the persistent current follow the dependence $I(\phi) \propto \sin \left(2 \pi \phi / \phi_{0}\right)[3]$.

the decay $L I \propto L^{-\alpha}$ is linear, with slope $-\alpha$. The CI and DMC data in figure 1 confirm the expected $[3,5]$ trend: The stronger the e-e interaction the shorter the system necessary to reach the $L I \propto L^{-\alpha}$ asymptotics. In figure 2 , where only the strongest e-e interaction is considered, the decay $L I \propto L^{-\alpha}$ exists already in rings with four electrons. To see this Luttinger-liquid feature in such few-electron system is a surprising result. Further, the CI and DMC data in figures 1 and 2 confirm that $\alpha$ obeys the RG formula (5). Finally, figure 3 shows, that the persistent current is a sine function of the magnetic flux also in the CI and DMC models .

We thank for the grant APVV-51-003505, VEGA grant 2/6101/27 and ESF project VCITE at IEESAS. L.M. thanks for support by NSF.

\section{References}

[1] J. Voit, Rep. Prog. Phys. 58, 977 (1995).

[2] Y. Imry, Introduction to Mesoscopic Physics (Oxford University Press, Oxford, UK, 2002).

[3] A. O. Gogolin, N. V. Prokof'ev, Phys. Rev. B 50, 4921 (1994).

[4] K. A. Matveev, D. Yue, L. I. Glazman, Phys. Rev. Lett. 71, 3351 (1993)

[5] V.Meden,U.Schollwöck,Phys.Rev.B67,035106 (2003).

[6] R. Nemeth and M. Mosko, cond-mat/0503146

[7] W. Foulkes, L. Mitas, R. J. Needs, and G. Rajagopal, Rev. Mod. Phys. 73, 33 (2001). 
[8] P. Vagner, M. Mosko, R. Nemeth, L. Wagner, L. Mitas, Physica E 32, 350 (2006). 